\title{
Plasma metabolomic analysis of human hepatocellular carcinoma: Diagnostic and therapeutic study
}

\author{
Yang Chen ${ }^{1, *}$, Jianyin Zhou ${ }^{2, *}$, Jinquan $\mathrm{Li}^{1}$, Jianghua Feng ${ }^{1}$, Zhong Chen ${ }^{1}$, Xiaomin Wang ${ }^{2}$ \\ ${ }^{1}$ Department of Electronic Science, Fujian Provincial Key Laboratory of Plasma and Magnetic Resonance, Xiamen University, \\ Xiamen, 361005, China \\ ${ }^{2}$ Department of Hepatobiliary and Pancreatic Surgery, Zhongshan Hospital, Xiamen University, Xiamen, 361004, China \\ *These authors contributed equally to this work
}

Correspondence to: Jianghua Feng, email: jianghua.feng@xmu.edu.cn Xiaomin Wang, email: wxm2203@xmu.edu.cn

Keywords: hepatocellular carcinoma, metabolic profiling, ${ }^{1} \mathrm{H}$-NMR spectroscopy, transcatheter arterial chemoembolization, surgery

Received: March 28, 2016

Accepted: May 23, 2016

Published: June 17, 2016

\section{ABSTRACT}

Many hepatocellular carcinoma (HCC) patients suffer from late stages when diagnosed, leading to dismal prospects for cure. Improving the diagnosis and treatment of HCC remains a challenge. In this work, NMR-based metabolomic techniques were used to investigate the metabolic alterations of HCC patients from different pathological backgrounds. Metabolic improvement of clinical surgical treatments or transcatheter arterial chemoembolization (TACE) for recurrent or metastatic HCC was also evaluated. HCC was characterized by enhanced lipid metabolism and high consumption in response to liver injury. Expectedly, higher consumption of glucose and lactate production in TACE group confirmed that recurrent or metastatic HCC is more active in citric acid cycle and oxidative phosphorylation. However, TACE or surgical treatments did not immediately improve the metabolic profiles of HCC patients. Combining multivariate statistical analyses with univariate $t$-test, a series of characteristic metabolites were identified and served as biomarkers for discrimination of HCC patients in different pathological backgrounds. The relative metabolic pathway analyses help to get insight into the underlying biochemical mechanism and extend clinical relevance. Furthermore, algorithm of support vector classification was used to identify HCC and control subjects, and diagnostic sensitivity and specificity reached to $100 \%$ and $81.08 \%$ respectively by receiver operating characteristic analysis. It is concluded that NMR-based metabolomic analysis of plasma can provide a powerful approach to discover diagnostic and therapeutic biomarkers, and subsequently contribute to clinical disease management.

\section{INTRODUCTION}

Human hepatocellular carcinoma (HCC), with the third highest mortality, is one of the most common malignant tumors in the world. Most deaths from liver diseases are attributed to HCC [1]. Even though more than half of the cases come from China, a dramatically increasing incidence of $\mathrm{HCC}$ in the world has recently been reported in developed countries such as France, Japan, the UK, and the USA [2]. As an aggressive tumor, $\mathrm{HCC}$ is largely occurring on individuals with a previous liver disease. Although it can be clinically detectable by tissue-based histopathological evaluation and/or bloodbased biochemical assays, notably by expression of alpha-fetoprotein (AFP) in the blood of advanced cases. However, these standard approaches may be unqualified and suffer from a lack of both sensitivity and specificity for early diagnosis [3]. Many patients have had advanced HCC stages when diagnosed and the prospects for cure are dismal. Consequently, there is an urgent need to seek new biomarkers for accurate diagnosis of HCC.

Recently, metabolomics has been proved to be a highly successful approach that is capable of detecting metabolic changes under different pathophysiological status [4]. By measuring changes in metabolite concentrations in the biological tissues or biofluids, the mutagenicity and progression of a disease can be determined and monitored $[5,6]$. Metabolomics based on nuclear magnetic resonance 
(NMR) spectroscopy or mass spectroscopy could be used to identify biomarkers for specific pathological or physiological status. Metabolomic analysis of human tissues and biofluids has been increasingly used to unveil metabolic alterations associated with different cancer types, such as breast [7], kidney [8], lung [9], prostate [10], and colorectal cancers [11]. In the case of liver diseases, a number of studies have been focusing on the metabolic profiling of tissues or biofluids. Shariff et al. and Wu et al. respectively reported that a set of urinary metabolites (creatinine, carnitine, creatine, and acetone, etc) can be used to identify metabolic changes associated with $\operatorname{HCC}[12,13]$ in Nigerian and Chinese populations. Apolito and colleagues performed tissue-based liquid chromatography-mass spectrometry to discriminate primary HCC from hepatic colorectal metastases via the changes of basic amino acids (arginine, citrulline, ornithine) [14]. Differential metabolites including alanine, leucine, and glucose have been identified to define hepatic tumorigenesis [15, 16]. Moreover, metabolic abnormalities have also been demonstrated by serum and plasma studies associated with the severity of liver disease. Fages et al. found sixteen metabolites in serum were significantly associated with HCC risk of a European prospective cohort [17]. Other studies show significant differences between compensated and decompensated cirrhosis, and between alcoholic cirrhosis and viral hepatitis [18-20]. The influence of hepatitis infection and potential liver damage were simultaneously assessed [21, 22]. To date, several NMR or mass spectrometry-based serum metabolomic studies have been conducted to study HCC $[17,19,20,23]$. And discriminatory metabolic alterations in HCC patients could draw a basic conclusion that altered mitochondrial respiration and glycolytic pathways lead to altered metabolic profiles in tumor cells [24, 25], but the identified metabolites are not concordant across these studies. Hence, these identified metabolites need to be further validated, not only that, global metabolic evaluation need to be simultaneously explored and studied to see alterations in the clinical treatment response or in the prognoses associated with recurrence or metastasis of HCC.

In this work, we present plasma metabolic profiling via ${ }^{1} \mathrm{H}$ NMR spectra of HCC patients from the different pathological backgrounds in comparison with healthy humans. Our aims are: (i) to depict the plasma metabolic characteristics of HCC patients for validation purpose; and (ii) to investigate potential metabolic alterations of postsurgical treatment and prognoses.

\section{RESULTS AND DISCUSSION}

\section{Discrimination of metabolic profiles between control and HCC patients}

A total of 168 spectra of plasma samples were acquired from different groups, including healthy controls $(n=60)$, HCC only subjects $(n=24)$, transcatheter arterial chemoembolization (TACE) for recurrence or metastasis of HCC subjects $(n=18)$, and surgery related HCC subjects ( $n=33$ for pre- and post-operative, respectively). The average NMR spectra of plasma samples from different pathological backgrounds are shown in Figure 1, and the resonance assignments are summarized in Table $\mathrm{S} 1$ in Supplemental Information. Plasma contains all of the low molecular weight metabolites including glucose, amino acids, organic acids, and metabolic intermediates and end-products. Some high molecular weight metabolites such as lipoproteins, fatty acids and phospholipids were also observed. NMR spectra of plasma under similar physiological conditions are highly reproducible, thus making them helpful for the diagnosis of diseased states.

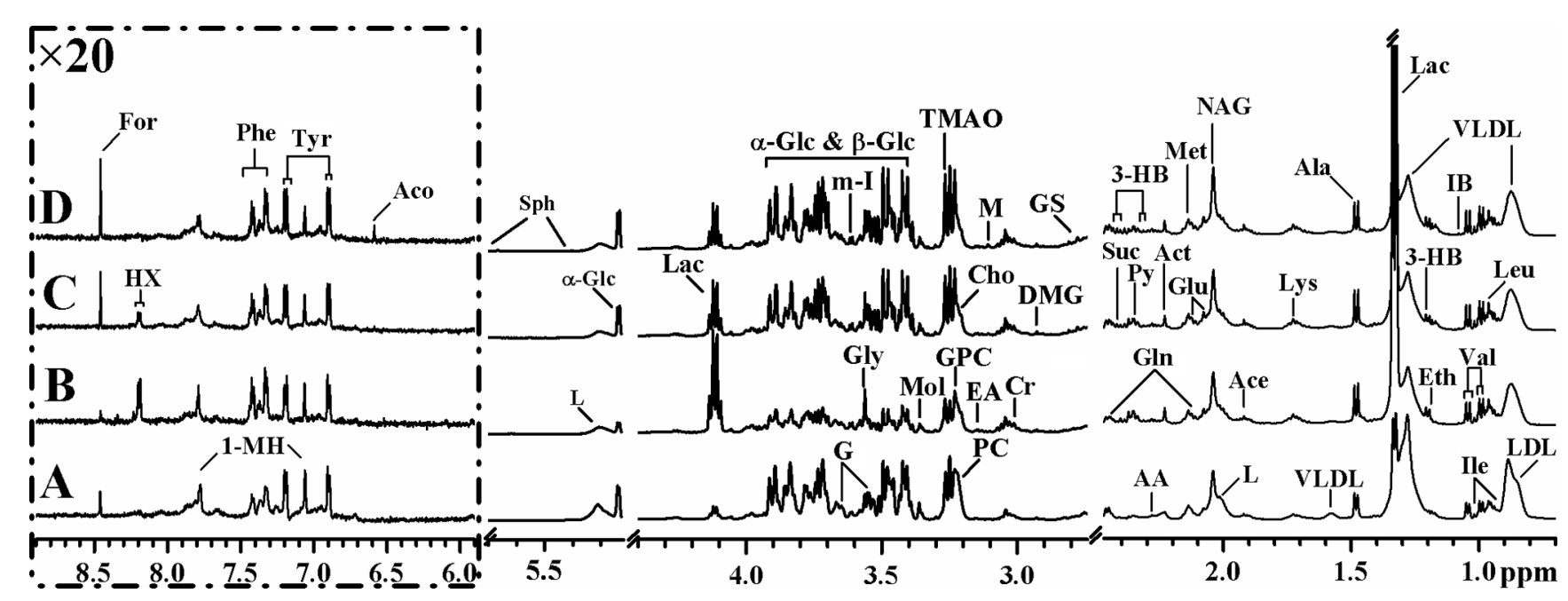

Figure 1: Average ${ }^{1} \mathbf{H}$ NMR spectra of plasma samples from different pathological backgrounds. (A) Healthy control; (B) TACE for recurrence or metastasis of HCC; (C) HCC only subject; (D) post-operative HCC subject. Keys for the assignments are shown in Table 2 and Table S1. 
Inspection of the ${ }^{1} \mathrm{H}$ NMR spectra reveals some obvious metabolic changes in HCC patients (with- or without- treatments) compared to healthy controls. For example, significantly decreased LDL, VLDL, and lipid concentrations and increased acetate and lactate concentrations are observed, which is consistent with previous reports of both rat model and human HCC patients $[19,26]$. Such observation is possibly due to enhanced lipid metabolism in response to liver injury caused by HCC. The increased levels of alanine, lactate, and pyruvate in intermediates of glycolysis and TCA cycle suggest high consumption of glucose by HCC in response to the stimulated aerobic glycolysis or the Warburg effect with conversion through pyruvate to alanine and lactate $[27,28]$. Notably, higher consumption of glucose and lactate production in TACE group than any other groups implied that recurrence or metastasis $\mathrm{HCC}$ is more active in conversion of glucose to lactic acid during mitochondrial citric acid cycle and oxidative phosphorylation. Besides, the levels of several free amino acids such as isoleucine, leucine, tyrosine, and valine have been shown to vary with different HCC groups. However, the more precise and detailed information need to be confirmed by multivariate analysis.

\section{Characteristic metabolites for HCC patients}

Principal component analysis (PCA) was performed on the plasma ${ }^{1} \mathrm{H}$ NMR data from different pair-wise groups to reveal trends and show clusters among the subjects. The PCA scores plots revealed an obvious separation between control $(n=60)$ and HCC patients ( $n=24$, without surgery or treatment) (Figure S1A), which implied abnormal metabolic pattern from HCC patients. Orthogonal projection to latent structure with discriminant analysis (OPLS-DA) was subsequently utilized to identify differential metabolites responsible for metabolic differences (Figure 2A). The reliability of OPLS-DA models could be evaluated by $\mathrm{Q}^{2}$ value and significant level of $P$ value. Usually larger $\mathrm{Q}^{2}$ value described better reliability. To determine statistical significance for these selected metabolites, relative concentrations were compared by Student's $t$-test analysis. As a result, seven selected metabolites were considered not significant and excluded in the final list of characteristic metabolites (Table 1). Lower levels of glucose, glycerophosphocholine, LDL, lipid, sphingosine, and VLDL, together with higher levels of lactate, and certain amino acids such as alanine, lysine, and valine were observed in HCC group than that of control, revealing high consumption of glucose in HCC subjects. Our results share some characteristic metabolites with some similar studies [17, 19, 25], including acetate, ethanol, glucose, etc. Meanwhile, some novel characteristic metabolites in the present work, such as alanine, hypoxanthine, sphingosine, etc., were also identified. It is suggestive that some kinds of populationrelated metabolic alteration are associated with HCC state, but a series of biological behaviors caused by high glucose consumption have become a common feature.

\section{HCC patients' metabolic responses to TACE and surgical treatments}

To further investigate metabolic improvement of clinical treatment and prognoses associated with recurrence or metastasis of $\mathrm{HCC}$, those patients who underwent TACE and surgical treatment were compared with control, respectively. As an intervention tool, treatment of TACE is often used in recurrent or metastatic liver cancer. Clearly, the TACE and control groups were separated into two classes along the first component on the PCA scores plots (Figure S1A in the Supplemental Information). The individual differences of TACE group seemed to be more significant than those of control group, suggesting different HCC states associated with recurrence or metastasis. However, the metabolic differences were still obvious (Figure 2B), implying unrecovered biological states of the TACE subjects. With regard to characteristic metabolites, higher levels of lactate, phenylalanine, and hypoxanthine and lower levels of glucose in TACE group gave the primary contribution to metabolic variations. Also, some other metabolites, including elevated levels of 3-hydoxybutyrate, acetone, pyruvate, succinate, isobutyrate, and the majority of amino acids and decreased levels of ethanol, lipid, LDL and VLDL, demonstrated in the plasma of TACE group (Figure 2B).

According to Table 1, fifteen of the selected metabolites were sifted as characteristic metabolites for TACE subjects. Notably, an abnormally high lactate concentration (approximately 4-fold higher than control) was observed. Actually clinical reports from the medical staff show these subjects most suffer from advanced tumor stages. Therefore, their pathological states could be confirmed by enhanced glucose consumption and lactate production [29]. The amount of characteristic metabolites of TACE group was less than that of HCC group. Unfortunately, we failed to establish a reliable OPLS-DA model (Figure 2C) between HCC and TACE groups since this model exhibited a quite low $\mathrm{Q}^{2}$ value and no significant differences $(P=1)$. Possibly due to no statistically significant differences as shown in the PCA scores plots (Figure S1A). So it is suggestive that metabolic differences between control and TACE groups are more significant than that between HCC and TACE, along with more common metabolic features but little differences. However, when we compare the effects of different treatments, we observed a big difference.

The surgical treatment did not significantly improve the metabolic profiles of HCC patients (Figure S1B and Figure 3A). According to the OPLS-DA model, many 
discriminatory metabolites were identified from postoperative group (Figure 3A). Even after the confirmation via $t$-test (Table 1), there are still 27 metabolites left as characteristic metabolites. Such result indicated that plasma metabolome still demonstrated increased glucose processing through glycolysis to pyruvate and other intermediates in TCA cycle, as well as lipid biosynthesis and glutaminolysis at post-surgery [30]. The similar result was also get from comparison of pre- and post-operative subjects, where mixed differentiation demonstrated no essential difference (Figure S1C and Figure 3B). Although lactate and phenylalanine are identified, further $t$-test eliminates the latter (Table 1). It is puzzling because the surgery is supposed to relieve the activity of cancer cells. A possible cause is the time point of blood sampling, since the majority of post-operative blood samples were collected in $24 \mathrm{~h}$ after surgery. It is obvious that only one day is deficient to suppress tumor activity.

Analysis of TACE and post-operative subjects was also undertaken to investigate treatment-induced metabolic alteration (Figure S1D and Figure 3C). Significant differentiation was achieved. A series of discriminating metabolites were identified (right panel of Figure 3C) and some of them were confirmed by $t$-test screening (Table 1). Notably, increased production of lactate and pyruvate were observed in both TACE and post-surgery plasma comparing with control, possibly due to declining pyruvate into the citric acid cycle in mitochondria [27]. Actually, different levels of glucose consumption reflect the activity of tumor, suggesting that recurrence or metastasis of HCC shows a stronger vitality.

\section{Metabolic pathways analysis}

A MATLAB-based tool was used to draw the map of relative biochemical pathways [31], and the custom subnetworks for HCC were created by using main substrateproduct pairs as defined by KEGG online database. In the HCC-related metabolic network (Figure 4), eleven biochemical pathways, including energy metabolism like glycolysis, biosynthesis of amino acids, metabolisms of amino acids, pyruvate, glycerolipid, sphingolipid, purine and butanoate, and TCA cycle, were involved in pathological changes of HCC patients.
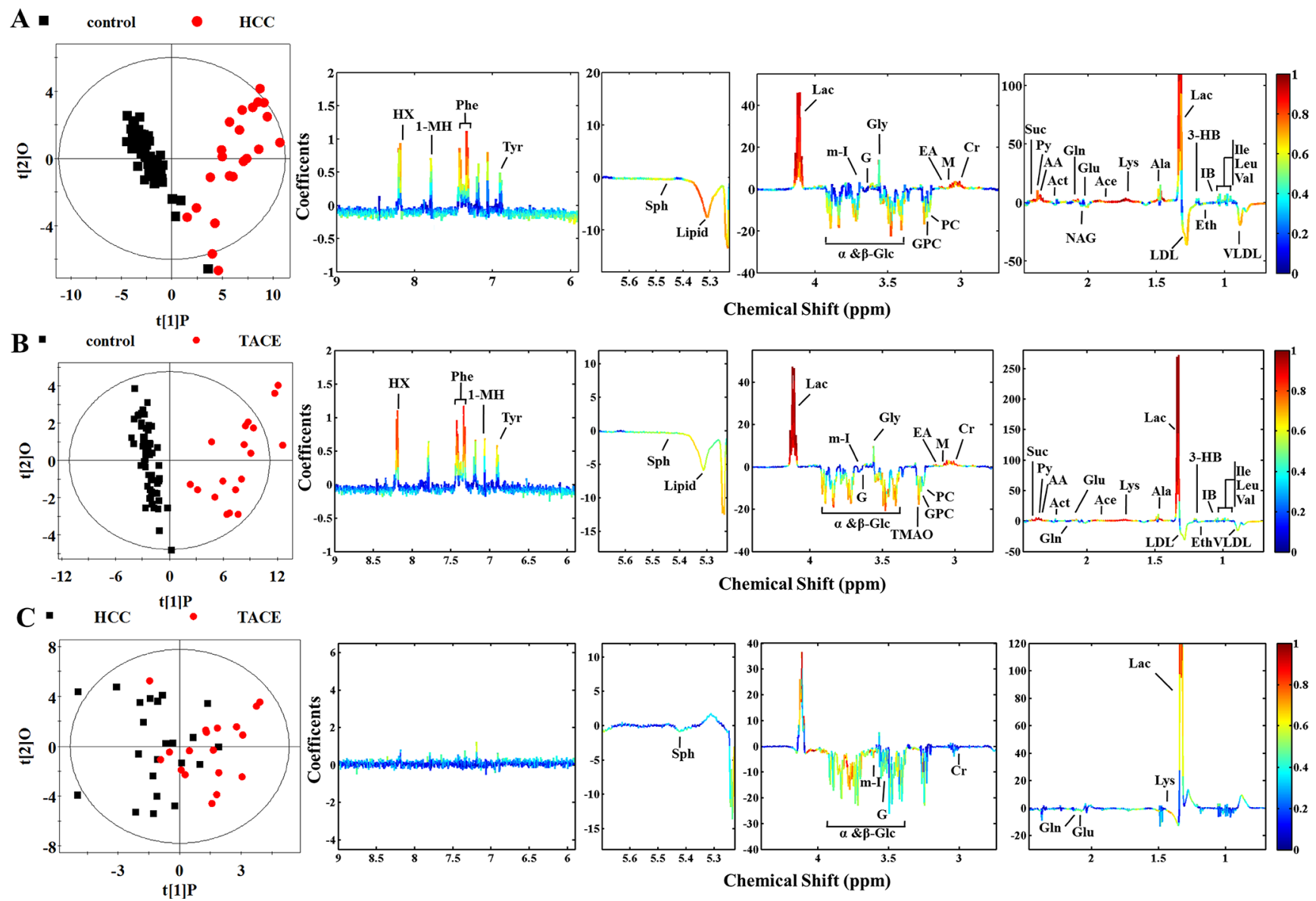

Figure 2: Scores and loading plots for determination of plasma metabolites responsible for the metabolic variations in different pair-wise pathological subjects via OPLS-DA analyses. (A) Control versus $H C C$ groups $\left(\mathrm{R}^{2} \mathrm{X}=52.2 \%, \mathrm{R}^{2} \mathrm{Y}=0.836\right.$, $\left.\mathrm{Q}^{2}=0.798, P<0.0001\right)$; (B) control versus TACE groups $\left(\mathrm{R}^{2} \mathrm{X}=58.5 \%, \mathrm{R}^{2} \mathrm{Y}=0.880, \mathrm{Q}^{2}=0.849, P<0.0001\right)$; (C) HCC versus TACE groups $\left(\mathrm{R}^{2} \mathrm{X}=48.1 \%, \mathrm{R}^{2} \mathrm{Y}=0.381, \mathrm{Q}^{2}=-0.041, P=1\right)$. 
Table 1: The relative concentrations of the characteristic metabolites in the plasma of HCC patients from different pathological backgrounds

\begin{tabular}{|c|c|c|c|c|c|c|}
\hline Metabolite & Abbr. & Control & $\mathrm{HCC}$ & TACE & Pre-surgery & Post-surgery \\
\hline low-density lipoprotein & LDL & $13.91 \pm 3.21^{*}$ & $9.51 \pm 1.93^{\mathrm{a}}$ & $9.82 \pm 2.40^{\mathrm{a}}$ & $9.74 \pm 2.77$ & $10.26 \pm 2.22^{\mathrm{a}}$ \\
\hline very low-density lipoprotein & VLDL & $9.94 \pm 4.04$ & $6.58 \pm 1.90^{\mathrm{a}}$ & $6.45 \pm 2.17^{\mathrm{a}}$ & $6.64 \pm 2.85$ & $6.40 \pm 2.22^{\mathrm{a}}$ \\
\hline Isoleucine & Ile & $1.03 \pm 0.13$ & $1.20 \pm 0.38^{\mathrm{a}}$ & $1.14 \pm 0.17$ & $1.19 \pm 0.20$ & $1.20 \pm 0.14$ \\
\hline Leucine & Leu & $1.15 \pm 0.16$ & $1.44 \pm 0.62^{\mathrm{a}}$ & $1.36 \pm 0.22$ & $1.29 \pm 0.29$ & $1.24 \pm 0.19^{\mathrm{c}}$ \\
\hline Isobutyrate & IB & $0.05 \pm 0.02$ & $0.08 \pm 0.05^{\mathrm{a}}$ & $0.08 \pm 0.02$ & $0.06 \pm 0.04$ & $0.06 \pm 0.03^{\mathrm{a}, \mathrm{b}}$ \\
\hline Ethanol & Eth & $0.42 \pm 0.23$ & $0.27 \pm 0.09^{\mathrm{a}}$ & $0.23 \pm 0.08^{\mathrm{a}}$ & $0.66 \pm 0.39$ & $0.60 \pm 0.31^{\mathrm{a}, \mathrm{b}}$ \\
\hline 3-Hydroxybutyrate & 3-HB & $0.99 \pm 0.16$ & $1.76 \pm 0.61^{\mathrm{a}}$ & $1.72 \pm 0.50^{\mathrm{a}}$ & $1.57 \pm 0.65$ & $1.57 \pm 0.86^{\mathrm{a}}$ \\
\hline Lactate & $\mathrm{Lac}$ & $7.90 \pm 1.57$ & $25.19 \pm 10.38^{\mathrm{a}}$ & $30.82 \pm 10.36^{\mathrm{a}}$ & $10.32 \pm 1.77$ & $11.60 \pm 3.09^{\mathrm{a}, \mathrm{b}, \mathrm{c}}$ \\
\hline Alanine & Ala & $1.13 \pm 0.20$ & $1.73 \pm 0.60^{\mathrm{a}}$ & $1.63 \pm 0.36^{\mathrm{a}}$ & $1.21 \pm 0.39$ & $1.12 \pm 0.28^{\mathrm{a}}$ \\
\hline Acetate & Ace & $0.16 \pm 0.05$ & $0.25 \pm 0.04$ & $0.24 \pm 0.05$ & $0.25 \pm 0.08$ & $0.24 \pm 0.08^{\mathrm{a}}$ \\
\hline Lipid & Lipid & $5.64 \pm 1.64$ & $3.93 \pm 0.73^{\mathrm{a}}$ & $3.96 \pm 0.90^{\mathrm{a}}$ & $4.21 \pm 1.13$ & $4.50 \pm 1.01^{\mathrm{a}}$ \\
\hline Glutamate & Glu & $2.93 \pm 0.45$ & $3.67 \pm 0.40$ & $3.29 \pm 0.47$ & $4.01 \pm 0.87$ & $3.74 \pm 0.65^{\mathrm{a}}$ \\
\hline Acetone & Act & $0.22 \pm 0.09$ & $0.29 \pm 0.14^{\mathrm{a}}$ & $0.33 \pm 0.19^{\mathrm{a}}$ & $0.27 \pm 0.15$ & $0.26 \pm 0.20^{\mathrm{a}}$ \\
\hline Acetoacetate & AA & $0.10 \pm 0.02$ & $0.13 \pm 0.03$ & $0.12 \pm 0.03$ & $0.14 \pm 0.06$ & $0.13 \pm 0.06^{\mathrm{a}, \mathrm{b}}$ \\
\hline Pyruvate & $\mathrm{Py}$ & $0.09 \pm 0.03$ & $0.43 \pm 0.32^{\mathrm{a}}$ & $0.33 \pm 0.16^{\mathrm{a}}$ & $0.19 \pm 0.12$ & $0.17 \pm 0.08^{\mathrm{a}, \mathrm{b}}$ \\
\hline Succinate & Suc & $0.05 \pm 0.02$ & $0.14 \pm 0.06^{\mathrm{a}}$ & $0.14 \pm 0.05^{\mathrm{a}}$ & $0.11 \pm 0.10$ & $0.11 \pm 0.09^{\mathrm{a}}$ \\
\hline Guanidinosuccinate & GS & $0.63 \pm 0.12$ & $0.68 \pm 0.19^{\mathrm{a}}$ & $0.67 \pm 0.17$ & $0.98 \pm 0.47$ & $1.05 \pm 0.53^{\mathrm{a}, \mathrm{b}}$ \\
\hline $\mathrm{N}, \mathrm{N}-$ Dimethylglycine & DMG & $0.06 \pm 0.03$ & $0.08 \pm 0.02$ & $0.08 \pm 0.02$ & $0.11 \pm 0.07$ & $0.12 \pm 0.08^{\mathrm{a}, \mathrm{b}}$ \\
\hline Creatine & $\mathrm{Cr}$ & $0.53 \pm 0.13$ & $0.75 \pm 0.15$ & $0.70 \pm 0.15$ & $0.78 \pm 0.19$ & $0.77 \pm 0.14^{c}$ \\
\hline Malonate & $\mathrm{M}$ & $0.05 \pm 0.03$ & $0.08 \pm 0.02$ & $0.08 \pm 0.02$ & $0.13 \pm 0.10$ & $0.16 \pm 0.14^{\mathrm{a}, \mathrm{b}, \mathrm{c}}$ \\
\hline Ethanolamine & EA & $0.12 \pm 0.06$ & $0.19 \pm 0.05$ & $0.21 \pm 0.05$ & $0.19 \pm 0.13$ & $0.21 \pm 0.16^{\mathrm{a}, \mathrm{b}}$ \\
\hline Phosphocholine & $\mathrm{PC}$ & $0.67 \pm 0.19$ & $0.54 \pm 0.14$ & $0.52 \pm 0.14$ & $0.55 \pm 0.16$ & $0.51 \pm 0.14^{\mathrm{a}}$ \\
\hline$\beta$-Glucose & $\beta$-Glc & $16.35 \pm 3.58$ & $9.57 \pm 6.06^{\mathrm{a}}$ & $7.29 \pm 4.30$ & $17.81 \pm 3.36$ & $17.10 \pm 2.38^{\mathrm{a}, \mathrm{b}}$ \\
\hline Trimethylamine $\mathrm{N}$-oxide & TMAO & $0.62 \pm 0.15$ & $0.56 \pm 0.20$ & $0.48 \pm 0.18$ & $0.91 \pm 0.24$ & $0.92 \pm 0.23^{\mathrm{a}}$ \\
\hline Methanol & Mol & $0.39 \pm 0.34$ & $0.19 \pm 0.05^{\mathrm{a}}$ & $0.21 \pm 0.10^{\mathrm{a}}$ & $0.31 \pm 0.15$ & $0.25 \pm 0.12^{\mathrm{a}}$ \\
\hline$\alpha$-Glucose & $\alpha$-Glc & $11.30 \pm 2.48$ & $6.49 \pm 3.90^{\mathrm{a}}$ & $4.96 \pm 2.70$ & $12.23 \pm 2.21$ & $11.61 \pm 1.40^{\mathrm{a}, \mathrm{b}}$ \\
\hline Glycerol & $\mathrm{G}$ & $2.63 \pm 1.00$ & $2.41 \pm 0.56^{\mathrm{a}}$ & $2.22 \pm 0.30^{\mathrm{a}}$ & $2.85 \pm 0.73$ & $2.74 \pm 0.38^{\mathrm{a}}$ \\
\hline Sphingosine & Sph & $0.09 \pm 0.06$ & $0.21 \pm 0.02^{\mathrm{a}}$ & $0.08 \pm 0.04^{\mathrm{a}}$ & $0.59 \pm 0.06$ & $0.18 \pm 0.08^{\mathrm{a}, \mathrm{b}}$ \\
\hline cis-Aconitate & Aco & $0.01 \pm 0.00$ & $0.01 \pm 0.01$ & $0.01 \pm 0.00^{\mathrm{a}}$ & $0.03 \pm 0.02$ & $0.04 \pm 0.01^{\mathrm{a}, \mathrm{b}}$ \\
\hline Tyrosine & Tyr & $0.14 \pm 0.04$ & $0.18 \pm 0.06^{\mathrm{a}}$ & $0.21 \pm 0.07^{\mathrm{a}}$ & $0.16 \pm 0.09$ & $0.19 \pm 0.08^{\mathrm{a}}$ \\
\hline 1-Methylhistidine & 1-MH & $0.11 \pm 0.05$ & $0.13 \pm 0.03^{\mathrm{a}}$ & $0.14 \pm 0.04$ & $0.09 \pm 0.09$ & $0.10 \pm 0.07$ \\
\hline Phenylalanine & Phe & $0.13 \pm 0.05$ & $0.33 \pm 0.11^{\mathrm{a}}$ & $0.36 \pm 0.12^{\mathrm{a}}$ & $0.19 \pm 0.17$ & $0.25 \pm 0.14^{\mathrm{a}}$ \\
\hline Hypoxanthine & $\mathrm{HX}$ & $0.01 \pm 0.00$ & $0.09 \pm 0.07^{\mathrm{a}}$ & $0.11 \pm 0.06^{\mathrm{a}}$ & $0.05 \pm 0.02$ & $0.04 \pm 0.02^{\mathrm{a}, \mathrm{b}}$ \\
\hline Formate & For & $0.04 \pm 0.01$ & $0.01 \pm 0.00$ & $0.01 \pm 0.00$ & $0.09 \pm 0.05$ & $0.10 \pm 0.05^{\mathrm{a}, \mathrm{b}}$ \\
\hline
\end{tabular}

${ }^{*}$ The relative concentrations of metabolites are presented as mean $\pm \mathrm{SD}$ of the integration value of the characteristic resonance of each metabolite.

Characteristic metabolite with $P<0.05$ versus: ${ }^{\mathrm{a}}$ control group, ${ }^{\mathrm{b}} \mathrm{TACE}$, and ${ }^{\mathrm{c}}$ preoperative subjects.

Furthermore, by extracting the common characteristic metabolites from HCC only, TACE, and surgery groups, the involved metabolic pathways composed the insensitive response to clinical treatments (Figure 5). In general, there are 12 common characteristic metabolites (lipid is not shown here) and 11 corresponding pathways are involved, which indicate insensitivity after TACE or surgical treatment. Increased levels of 3-hydroxybutyrate involved 
in butanoate metabolism, indicate upregulation of the synthesis of ketone bodies. This results in the accumulation of acetyl-CoA and sequentially alters the TCA cycle, and purine, pyruvate metabolisms $[32,33]$. Elevated alanine, phenylalanine, and tyrosine levels in HCC and TACE groups indicate that abnormal biosynthesis of amino acid metabolism still occurs even after clinical treatments. Others found these aromatic amino acids to be elevated in HCC patients with liver failure [19, 34], and their increases were attributed to high levels of biosynthesis precursors $[35,36]$. Therefore, TACE or surgical treatment may fail to inhibit perturbation involved in tyrosine and phenylalanine metabolisms. In addition, lactate, which is also involved in pyruvate metabolism, is elevated in all HCC groups with different degrees (Figure 5). This finding represents a major difference in the interconversion of lactate and pyruvate. According to these features, pyruvate metabolism of these patients may have been disrupted in some way, but the treatments did not improve the disorders. This metabolic network suggested that these biochemical pathways should be targeted for the therapeutic purpose of HCC patients in clinical management.

\section{Classification of HCC patients by plasma- derived characteristic metabolites}

The characteristic metabolites were fed back to explore the ability in classifying HCC and control subjects combining with support vector machines (SVM) via LIBSVM package. We randomly extracted 50\% samples from each group as training sets and remainders were used as validation sets. The receiver operating characteristic (ROC) curve of support vector classification (SVC)-based prediction is shown in Figure 6, along with the overall performances including area under the curve (AUC) and the corresponding diagnostic sensitivity and specificity.

The AUC for a perfect case will be 1.000. No control sample was misclassified, making the sensitivity reach $100 \%$ while specificity is only $81.08 \%$. Besides, these results showed that SVC algorithm based on characteristic metabolites data sets performed robustly and again HCC patients have different metabolic profiles accountable for their biological properties. Moreover, the potential and extend of transforming characteristic metabolites to disease biomarkers still need to be tested in future clinical applications.
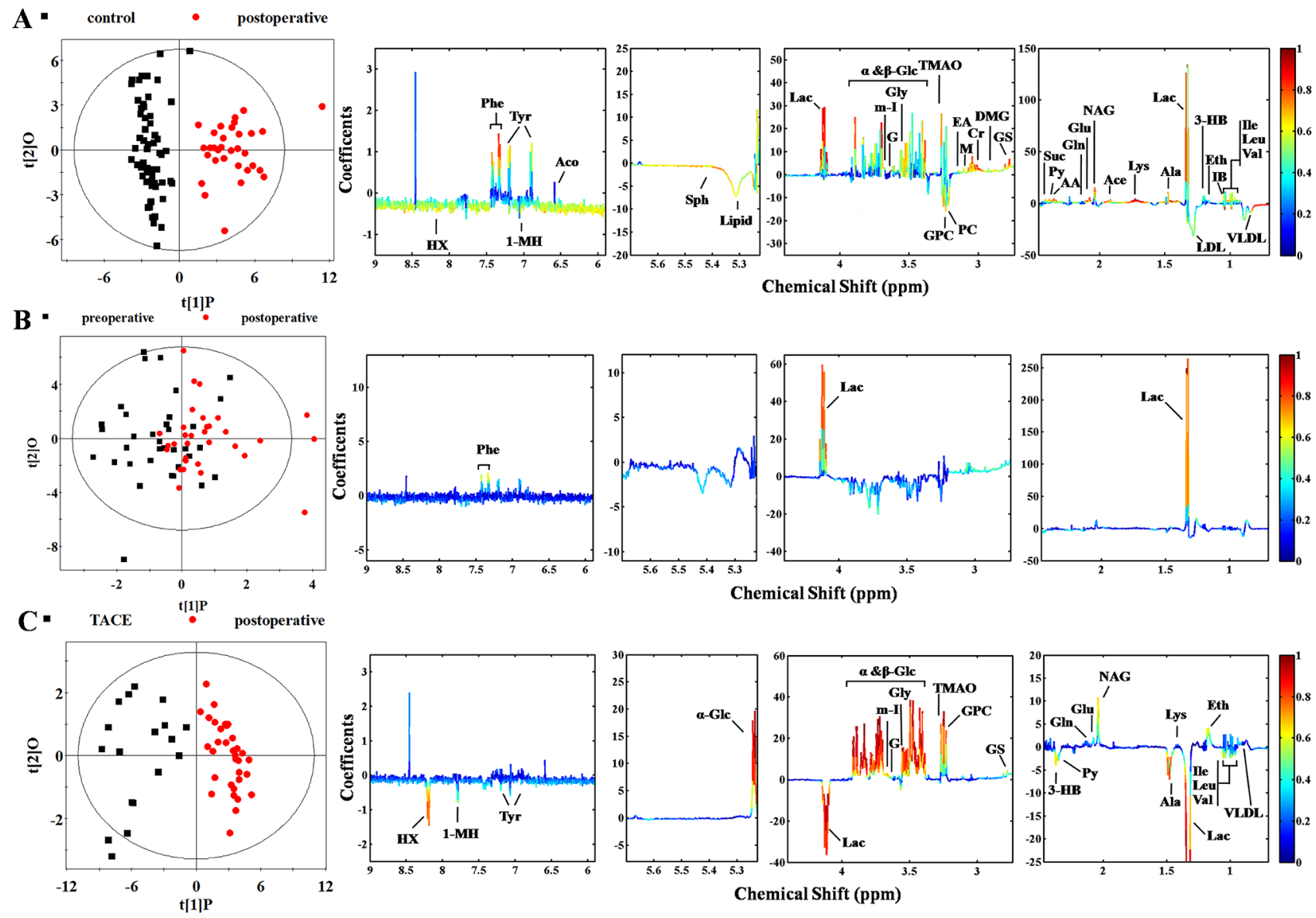

Figure 3: Score and loading plots for determination of metabolites responsible for plasma from groups of control, surgery, and TACE via OPLS-DA analyses. (A) Control versus post-operative group $\left(\mathrm{R}^{2} \mathrm{X}=48.2 \%, \mathrm{R}^{2} \mathrm{Y}=0.841, \mathrm{Q}^{2}=0.792\right.$, $P<0.0001)$; (B) pre- versus post-operative group $\left(\mathrm{R}^{2} \mathrm{X}=29.3 \%, \mathrm{R}^{2} \mathrm{Y}=0.298, \mathrm{Q}^{2}=-0.150, P=1\right)$; (C) TACE versus post-operative group $\left(\mathrm{R}^{2} \mathrm{X}=45.7 \%, \mathrm{R}^{2} \mathrm{Y}=0.837, \mathrm{Q}^{2}=0.713, P<0.0001\right)$. 


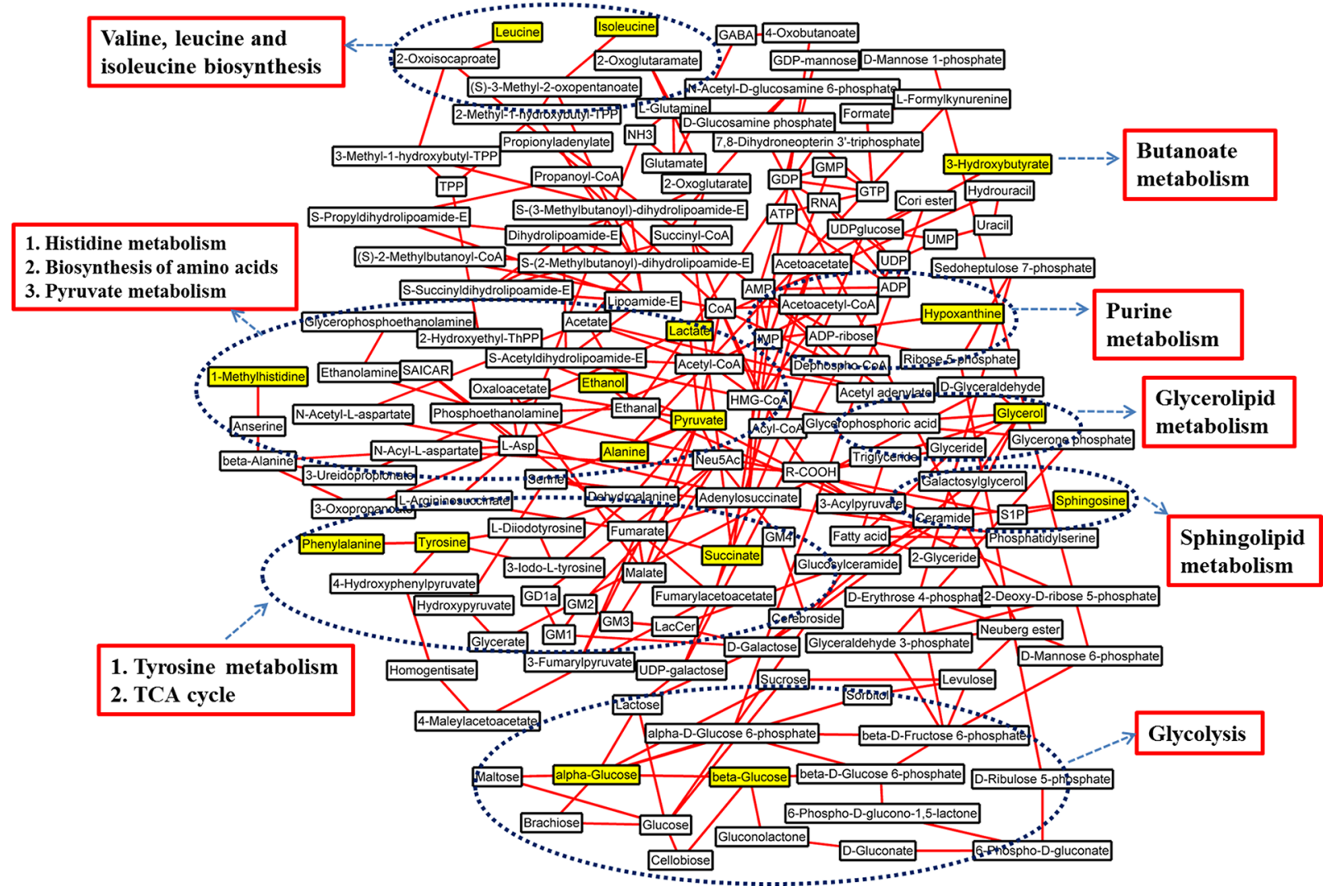

Figure 4: Schematic diagram of human HCC metabolic pathways. The metabolites in yellow backgrounds are the characteristic metabolites of HCC patients without surgery or TACE. The corresponding metabolic pathways are demonstrated in the dark blue ellipse and the names are identified by red box.

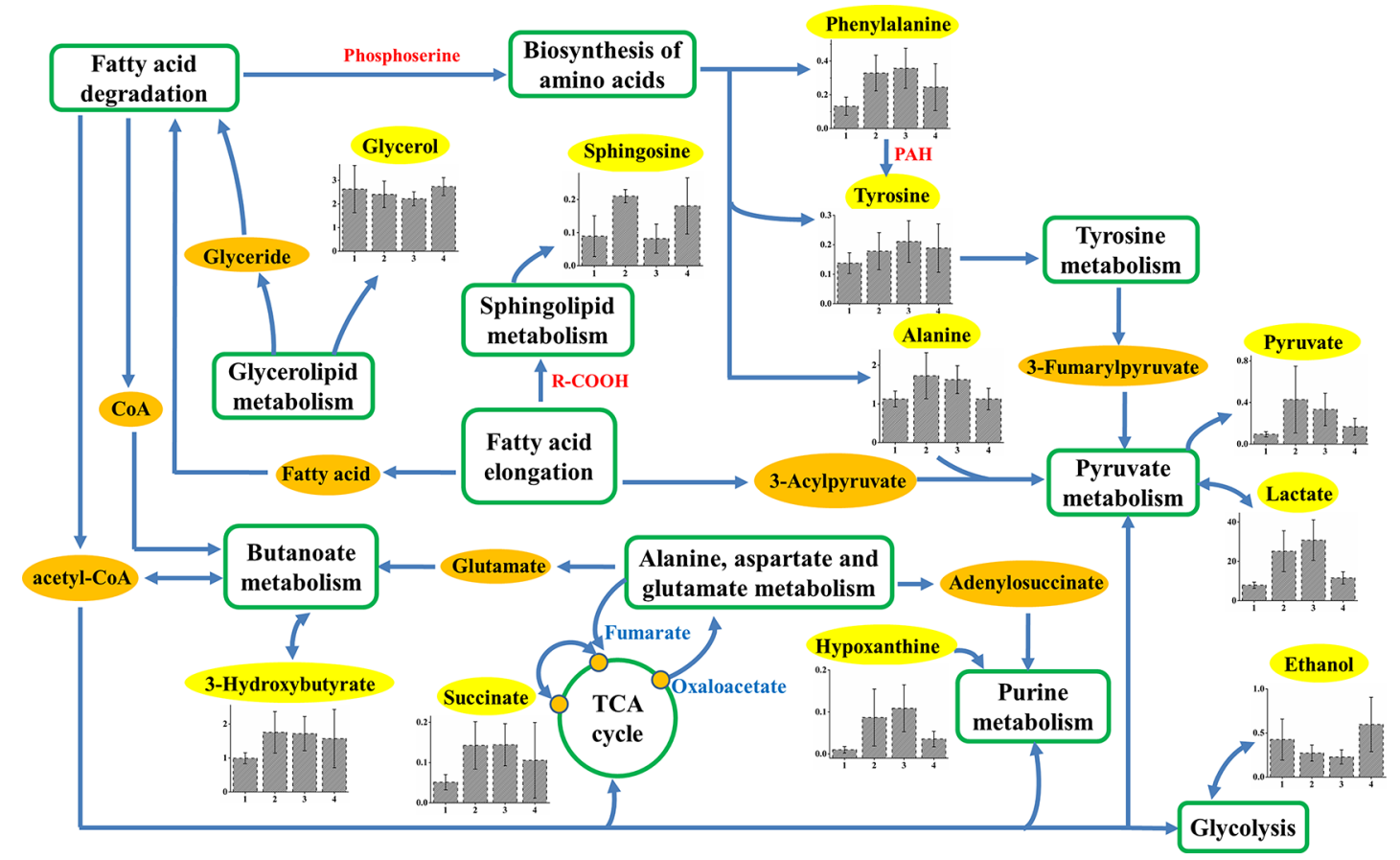

Figure 5: Schematic diagram of human HCC metabolic pathways that are not significant response to TACE or surgical treatments. Relative levels of characteristic compounds within these pathways are also shown. 1,2, 3, and 4 stand for plasma samples taken from control, HCC only, TACE, and surgery groups, respectively. The metabolites in yellow backgrounds are the common characteristic metabolites in HCC-related plasma samples, and the corresponding metabolic pathways are demonstrated in the green box or circle. 


\section{MATERIALS AND METHODS}

\section{Ethics statement and clinical subjects}

This study was approved by the Ethics committee of Xiamen University. The human plasma samples were used in accordance with the guidelines of Zhongshan Hospital Xiamen University. We recruited 57 patients with HCC (33 patients underwent surgical treatment), 18 patients underwent TACE (for recurrence or metastasis of HCC), and 60 healthy control subjects at the department of hepatobiliary surgery from April 2012 to May 2014. Following informed consent obtained from each subject, patients needed to fulfill the following inclusion criteria: (1) biopsy-proven HCC; (2) no infection by the human immunodeficiency hepatitis $\mathrm{C}$ viruses. HCC patients were diagnosed according to histological evidence or the combination of imaging techniques that showed this morphologic aspect plus an AFP level of $400 \mathrm{ng} / \mathrm{mL}$ or more. And the clinical information such as age, gender, and some important biochemical indexes for HCC patients and healthy subjects was presented in Table 2. The blood AFP, bilirubin and three enzyme levels correlating to liver function were measured on all subjects. The AFP levels were significantly higher in $\mathrm{HCC}$ than in control. Besides, associated HBV antibody was also noted in HCC patients.

\section{Plasma sample collection and preparation}

Venous blood samples were preprandially collected in the morning during routine clinical blood work using heparin tubes or citrate-rinsed tubes. During this procedure, blood samples from HCC patients underwent surgical treatment were collected before and after surgical treatment, respectively. Plasma was separated by centrifugation at $1,000 \mathrm{~g}$ at $4^{\circ} \mathrm{C}$ for $10 \mathrm{~min}$ and then immediately stored at $-80^{\circ} \mathrm{C}$ until further analyses. Before NMR analyses, the plasma samples were thawed at room temperature, and $400 \mu \mathrm{L}$ of aliquots were mixed with $200 \mu \mathrm{L}$ of $\mathrm{D}_{2} \mathrm{O}$-prepared phosphate-buffered saline (PBS, $\mathrm{pH}=7.4)$ and centrifuged at $10,000 \mathrm{~g}$ for $10 \mathrm{~min}$ at $4^{\circ} \mathrm{C}$. Subsequently, $550 \mu \mathrm{L}$ of the supernatant was transferred to a $5 \mathrm{~mm}$ NMR tube, and NMR acquisition was immediately performed.

\section{${ }^{1}$ H NMR spectroscopy}

The ${ }^{1} \mathrm{H}$ NMR measurements of plasma samples were performed using a $500 \mathrm{MHz}$ Varian NMR spectrometer equipped with a triple resonance probe, operating at a ${ }^{1} \mathrm{H}$ frequency of $499.74 \mathrm{MHz}$. The experimental temperature was set to $293 \mathrm{~K}$ and the $90^{\circ}$ pulse length was calibrated individually for each sample. Standard 1D ${ }^{1} \mathrm{H}$ spectra were acquired with a Carr-Purcell-Meiboom-Gill (CPMG)

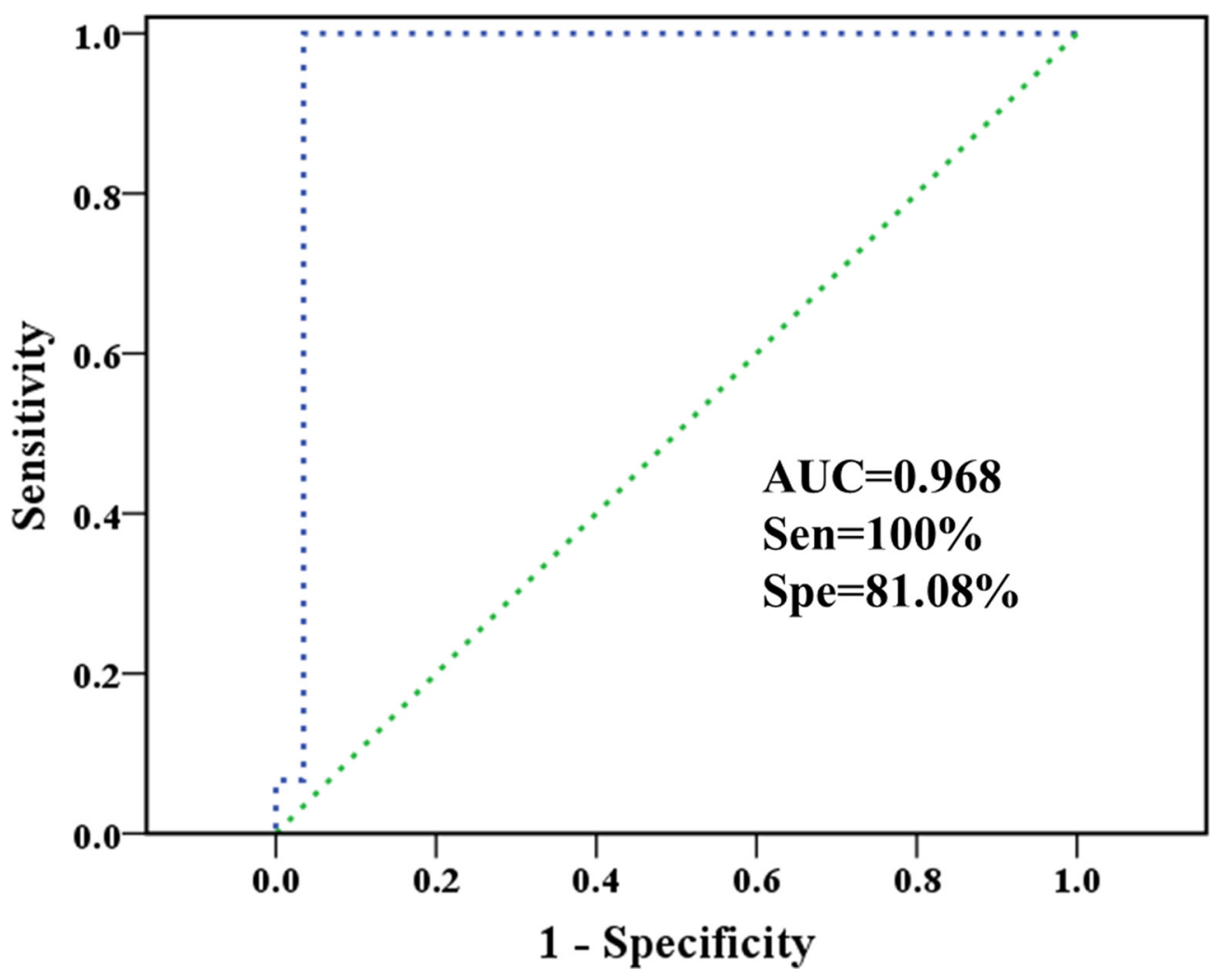

Figure 6: Classification of healthy controls and HCC patients based on plasma-derived characteristic metabolites. The ROC curve of SVC classifier, as well as the sensitivity, specificity, AUC, and diagnostic reference line are shown. 
Table 2: Clinical information for healthy humans and HCC patients

\begin{tabular}{|c|c|c|c|c|c|}
\hline Parameters & Control & $\mathrm{HCC}$ & TACE & Pre-surgery & Post-surgery \\
\hline Number & 60 & 24 & 18 & 33 & 33 \\
\hline Age & $58.5 \pm 11.0$ & $57.3 \pm 3.3$ & $60.5 \pm 8.7$ & $50.1 \pm 4.2$ & $50.1 \pm .2$ \\
\hline Male/Female & $36 / 24$ & $20 / 4$ & $15 / 3$ & $27 / 6$ & $27 / 6$ \\
\hline AFP value $(\mathrm{ng} / \mathrm{mL})$ & $22 \pm 1.1$ & $463.2 \pm 199.8$ & $55387.5 \pm 5399.5$ & $62105.4 \pm 2840.6$ & $15865.3 \pm 1103.9$ \\
\hline HBsAg (positive \%) & - & 100 & 89 & 50 & 50 \\
\hline$\overline{A L P}(\mathrm{IU} / \mathrm{L})$ & $72.0 \pm 18.3$ & $154.3 \pm 20.3$ & $124.8 \pm 18.4$ & $152.5 \pm 22.3$ & $129.8 \pm 17.4$ \\
\hline ALT (IU/L) & $18.0 \pm 6.4$ & $73.6 \pm 11.4$ & $97.1 \pm 23.8$ & $120.0 \pm 23.1$ & $56.5 \pm 9.7$ \\
\hline AST (IU/L) & $22.0 \pm 10.4$ & $74.5 \pm 13.3$ & $154.2 \pm 54.0$ & $151.2 \pm 58.7$ & $81.9 \pm 13.2$ \\
\hline D-BIL $(\mu \mathrm{mol} / \mathrm{L})$ & $2.5 \pm 1.2$ & $7.1 \pm 1.2$ & $11.0 \pm 4.4$ & $12.9 \pm 4.7$ & $5.9 \pm 0.8$ \\
\hline T-BIL ( $\mu \mathrm{mol} / \mathrm{L})$ & $9.2 \pm 3.2$ & $21.6 \pm 4.9$ & $14.6 \pm 2.4$ & $22.5 \pm 5.3$ & $14.3 \pm 2.2$ \\
\hline
\end{tabular}

Data are presented as mean \pm SE.

Abbreviations: AFP, alpha-fetoprotein; HBsAg, hepatitis B surface antigen; ALP, alkaline phosphatase; ALT, alanine aminotransferase; AST, aspartate transaminase; D-BIL, direct bilirubin; T-BIL, total bilirubin.

spin-echo pulse sequence with relaxation time of $2 \mathrm{~s}$ and acquisition time of $1 \mathrm{~s}$. A total of 128 scans with a spectral width of $10,000 \mathrm{~Hz}$ were collected into $64 \mathrm{~K}$ data points for all NMR experiments.

\section{Data preprocessing and pattern recognition}

All free induction decays (FIDs) were multiplied by a $1.0 \mathrm{~Hz}$ exponential line broadening factor prior to Fourier Transformation. The collected NMR spectra were manually phased and baseline corrected using the software MestReNova (version: 8.1.2, Mestrelab Research S.L., Spain) and referenced to the $\mathrm{CH}_{3}$ resonance of lactate at $\delta 1.33$. The peaks of metabolites observed in the plasma ${ }^{1} \mathrm{H}$ NMR spectra were assigned with reference to published data $[36,37]$ and confirmed by HMDB database [38]. The regions of $\delta 2.47-2.74, \delta 4.40-5.30$, and $\delta 5.70-5.90$ were excluded to remove the effects of citrate, urea and variation in residual water. Subsequently, the spectra were divided into regions of $0.002 \mathrm{ppm}$ and integrated in the region of $0.50-9.00 \mathrm{ppm}$. To account for variations in sample concentration, the spectra were normalized to the total sum of the spectrum before multivariate statistical analysis.

Protocols for statistical analysis have been described previously in full by Li et al. [39]. Briefly, PCA and OPLSDA were carried out using SIMCA-P + v14.0 (Umetrics, Sweden). Data used in PCA were mean-centered scaled while in OPLS-DA were Pareto scaled. The optimal number of orthogonal components for building OPLS-DA models was selected using a 15 -fold cross validation procedure. The goodness of fit and prediction parameters of OPLS-DA models, $\mathrm{R}^{2}$ and $\mathrm{Q}^{2}$ were calculated, and the corresponding probability ( $P$-value) of significant difference between the pair-wise groups were obtained by CV-ANOVA.
Visualization of results was based on score plots of the first two components, and a correlation coefficient of $|r|>0.45$ was used as the cut-off value for determination of characteristic metabolites with significance level of $P<0.05$ according to Student's $t$-test analysis.

Finally, the associative characteristic metabolites were fed back to identify HCC and control subjects by combining with SVM via LIBSVM package (Machine Learning and Data Mining Group) [40]. Since only about a dozen of metabolites were involved, SVM algorithm proposed by Vapnik was suitable for such small-sample problems [41]. The task of LIBSVM was SVC algorithm. Such algorithm aims to construct optimal hyper-plane in a higher dimensional space that maximal margin two classes according to the input data sets. The type is C_SVC with kernel function of the radial basis function. Automatic optimization parameters together with leave-one-out cross-validation were utilized to predict unknown samples and evaluate the reliability of SVC models.

\section{CONCLUSIONS}

In this study, NMR-based metabolomic techniques were used to identify the characteristic metabolic profiles of plasma from HCC patients in different pathological backgrounds. High consumption of glucose was revealed in HCC patients along with large amount of intermediates and end-products from aerobic glycolysis. Most of the characteristic metabolites were consistent with previous studies except the addition of some novel discoveries. A series of biological behaviors caused by high glucose consumption have become a common feature in HCC patients. Patients underwent TACE seem to be more discriminatory from control than other HCC patients, which is consistent with that TACE treatment was targeted 
at advanced subjects in clinical practice. The key response of surgery was revealed by an overall increase in energy metabolism. However, the TACE and surgical treatments didn't immediately induce obvious improvement in metabolic profiles. The corresponding metabolic pathway analysis vastly extends clinical relevance and effects of our proposed biomarkers. We are also aware that these results should be validated by a larger cohort of samples, and that external validations are essential to test the clinical validity of characteristic metabolites-derived classification models. The short interval of blood sampling after surgery is likely to expose one of the limitations of this study, and the confirmation should be contained in further work.

\section{ACKNOWLEDGMENTS AND FUNDING}

This work was supported by the National Natural Science Foundation of China (81272581), Science Research Foundation of Ministry of Health \& United Fujian Provincial Health and Education Project for Tackling the Key Research (WKJ-FJ-05), the Fundamental Research Funds for Xiamen University (201412G012), the Project of Natural Science Foundation of Fujian Province (2015J01541), and the Project funding for the training of young talents in the health system of Fujian Province (2014-ZQN-JC-42).

\section{CONFLICTS OF INTEREST}

The authors declare that there is no conflicts of interest.

\section{REFERENCES}

1. Dhanasekaran R, Limaye A, Cabrera R. Hepatocellular carcinoma: current trends in worldwide epidemiology, risk factors, diagnosis, and therapeutics. Hepat Med. 2012; 4:19-37.

2. El-Serag HB, Rudolph KL. Hepatocellular carcinoma: epidemiology and molecular carcinogenesis. Gastroenterology. 2007; 132:2557-2576.

3. Forner A, Bruix J. Biomarkers for early diagnosis of hepatocellular carcinoma. Lancet Oncol. 2012; 13:750-751.

4. Zhang A, Sun H, Yan G, Han Y, Ye Y, Wang X. Urinary metabolic profiling identifies a key role for glycocholic acid in human liver cancer by ultra-performance liquidchromatography coupled with high-definition mass spectrometry. Clin Chim Acta. 2013; 418:86-90.

5. Beyoğlu D, Imbeaud S, Maurhofer O, Bioulac-Sage P, Zucman-Rossi J, Dufour JF, Idle JR. Tissue metabolomics of hepatocellular carcinoma: tumor energy metabolism and the role of transcriptomic classification. Hepatology. 2013; 58:229-238.

6. Duarte IF, Gil AM. Metabolic signatures of cancer unveiled by NMR spectroscopy of human biofluids. Prog Nucl Magn Reson Spectrosc. 2012; 62:51-74.
7. Asiago VM, Alvarado LZ, Shanaiah N, Gowda GA, OwusuSarfo K, Ballas RA, Raftery D. Early detection of recurrent breast cancer using metabolite profiling. Cancer Res. 2010; 70:8309-8318.

8. Lin L, Yu Q, Yan X, Hang W, Zheng J, Xing J, Huang B. Direct infusion mass spectrometry or liquid chromatography mass spectrometry for human metabonomics? A serum metabonomic study of kidney cancer. Analyst. 2010; 135:2970-2978.

9. Rocha CM, Carrola J, Barros AS, Gil AM, Goodfellow BJ, Carreira IM, Bernardo J, Gomes A, Sousa V, Carvalho L, Duarte IF. Metabolic signatures of lung cancer in biofluids: NMR-based metabonomics of blood plasma. J Proteome Res. 2011; 10:4314-4324.

10. Lokhov PG, Dashtiev MI, Bondartsov LV, Lisitsa AV, Moshkovskii SA, Archakov AI. Metabolic fingerprinting of blood plasma from patients with prostate cancer. Biochemistry Supp. 2010; 4:37-41.

11. Qiu Y, Cai G, Su M, Chen T, Zheng X, Xu Y, Ni Y, Zhao A, Xu LX, Cai S, Jia W. Serum metabolite profiling of human colorectal cancer using GC-TOFMS and UPLCQTOFMS. J Proteome Res. 2009; 8:4844-4850.

12. Shariff MI, Ladep NG, Cox IJ, Williams HR, Okeke E, Malu A, Thillainayagam AV, Crossey MM, Khan SA, Thomas HC, Taylor-Robinson SD. Characterization of urinary biomarkers of hepatocellular carcinoma using magnetic resonance spectroscopy in a Nigerian population. J Proteome Res. 2010; 9:1096-1103.

13. Wu H, Xue R, Dong L, Liu T, Deng C, Zeng H, Shen X. Metabolomic profiling of human urine in hepatocellular carcinoma patients using gas chromatography/mass spectrometry. Anal Chim Acta. 2009; 648:98-104.

14. Oceania A, Daniela G, Monica G, Debora P, Dominique M, Rosa C, Francesco I, Giuseppe P, Giuseppe C, Andrea M, Gaetano C. Basic amino acids and dimethylarginines targeted metabolomics discriminates primary hepatocarcinoma from hepatic colorectal metastases. Metabolomics. 2014; 10:1026-1035.

15. Yang Y, Li C, Nie X, Feng X, Chen W, Yue Y, Tang H, Deng F. Metabonomic studies of human hepatocellular carcinoma using high-resolution magic-angle spinning ${ }^{1} \mathrm{H}$ NMR spectroscopy in conjunction with multivariate data analysis. J Proteome Res. 2007; 6:2605-2614.

16. Debora P, Dominique M, Matteo S, Oceania A, Rosa C, Giuseppe C, Francesco I, Giuseppe P, Gaetano C, Andrea M. Monitoring liver alterations during hepatic tumorigenesis by NMR profiling and pattern recognition. Metabolomics. 2010; 6:405-416.

17. Fages A, Duarte-Salles T, Stepien M, Ferrari P, Fedirko V, Pontoizeau C, Trichopoulou A, Aleksandrova K, Tjønneland A, Olsen A, Clavel-Chapelon F, Boutron-Ruault MC, Severi G. Metabolomic profiles of hepatocellular carcinoma in a European prospective cohort. BMC Med. $2015 ; 13: 242$.

18. Qi SW, Tu ZG, Peng WJ, Wang LX, Ou-Yang X, Cai AJ, Dai Y. ${ }^{1} \mathrm{H}$ NMR-based serum metabolic profiling in 
compensated and decompensated cirrhosis. World J Gastroenterol. 2012; 18:285-290.

19. Gao H, Lu Q, Liu X, Cong H, Zhao L, Wang H, Lin D. Application of ${ }^{1} \mathrm{H}$ NMR-based metabonomics in the study of metabolic profiling of human hepatocellular carcinoma and liver cirrhosis. Cancer Sci. 2009; 100:782-785.

20. Nahon P, Amathieu R, Triba MN, Bouchemal N, Nault JC, Ziol M, Seror O, Dhonneur G, Trinchet JC, Beaugrand M, Le Moyec L. Identification of serum proton NMR metabolomic fingerprints associated with hepatocellular carcinoma in patients with alcoholic cirrhosis. Clin. Cancer Res. 2012; 18:6714-6722.

21. Amathieu $R$, Triba $M N$, Nahon $P$, Bouchemal $N$, Kamoun W, Haouache H, Trinchet JC, Savarin P, Le Moyec L, Dhonneur G. Serum ${ }^{1} \mathrm{H}-\mathrm{NMR}$ metabolomic fingerprints of acute-on-chronic liver failure in intensive care unit patients with alcoholic cirrhosis. PLoS One. 2014; 9:e89230.

22. Amathieu R, Nahon P, Triba M, Bouchemal N, Trinchet JC, Beaugrand M, Dhonneur G, Le Moyec L. Metabolomic approach by ${ }^{1} \mathrm{H}$ NMR spectroscopy of serum for the assessment of chronic liver failure in patients with cirrhosis. J Proteome Res. 2011; 10:3239-3245.

23. Liu Y, Hong Z, Tan G, Dong X, Yang G, Zhao L, Chen X, Zhu Z, Lou Z, Qian B, Zhang G, Chai Y. NMR and LC/MSbased global metabolomics to identify serum biomarkers differentiating hepatocellular carcinoma from liver cirrhosis. Int J Cancer. 2014; 135:658-668.

24. Warburg O, Posener K, Negelein E. Uber den Stoffwechsel der Carcinomzelle. Biochem Zeitschr. 1924; 152:309-344.

25. Shariff MI, Gomaa AI, Cox IJ, Patel M, Williams HR, Crossey MM, Thillainayagam AV, Thomas HC, Waked I, Khan SA, Taylor-Robinson SD. Urinary metabolic biomarkers of hepatocellular carcinoma in an Egyptian population: a validation study. J Proteome Res. 2011; 10:1828-1836.

26. Zhao WD, Guan S, Zhou KR Li H, Peng WJ, Tang F, Chen ZW. In vivo detection of metabolic changes by ${ }^{1} \mathrm{H}-\mathrm{MRS}$ in the DEN-induced hepatocellular carcinoma in Wistar rat. J Cancer Res Clin Oncol. 2005; 131:597-602.

27. Darpolor MM, Yen YF, Chua MS, Xing L, ClarkeKatzenberg RH, Shi W, Mayer D, Josan S, Hurd RE, Pfefferbaum A, Senadheera L, So S, Hofmann LV, et al. In vivo MRSI of hyperpolarized [1-(13)C]pyruvate metabolism in rat hepatocellular carcinoma. NMR Biomed. 2011; 24:506-513.

28. Gatenby RA, Gillies RJ. Why do cancers have high aerobic glycolysis? Nat Rev Cancer. 2004; 4:891-899.

29. Brauer HA, Makowski L, Hoadley KA, CasbasHernandez $\mathrm{P}$, Lang LJ, Romàn-Pèrez E, D'Arcy $M$, Freemerman AJ, Perou CM, Troester MA. Impact of tumor microenvironment and epithelial phenotypes on metabolism in breast cancer. Clin Cancer Res. 2013; 19:571-585.

30. Hatziapostolou M, Polytarchou C, Iliopoulos D. miRNAs link metabolic reprogramming to oncogenesis. Trends Endocrinol Metab. 2013; 24:361-373.

31. Posma JM, Robinette SL, Holmes E, Nicholson JK. MetaboNetworks, an interactive Matlab-based toolbox for creating, customizing and exploring sub-networks from KEGG. Bioinformatics. 2014; 30:893-895.

32. Tisdale MJ. Cancer cachexia: metabolic alterations and clinical manifestations. Nutrition. 1997; 13:1-7.

33. Zhang J, Wei S, Liu L, Nagana Gowda GA, Bonney P, Stewart J, Knapp DW, Raftery D. NMR-based metabolomics study of canine bladder cancer. Biochim Biophys Acta. 2012; 1822:1807-1814.

34. Kalhan SC, Guo L, Edmison J, Dasarathy S, McCullough AJ, Hanson RW, Milburn M. Plasma metabolomic profile in nonalcoholic fatty liver disease. Metabolism. 2011; 60:404-413.

35. Munshi SU, Taneja S, Bhavesh NS, Shastri J, Aggarwal R, Jameel S. Metabonomic analysis of hepatitis E patients shows deregulated metabolic cycles and abnormalities in amino acid metabolism. J Viral Hepat. 2011; 18:e591-602.

36. Xu J, Jiang H, Li J, Cheng K-K, Dong J, Chen Z. ' $\mathrm{H}$ NMRbased metabolomics investigation of copper-laden rat: a model of Wilson's disease. PLoS One. 2015; 10:e0119654.

37. Holmes E, Foxall PJD, Spraul M, Farrant RD, Nicholson JK, Lindon JC. $750 \mathrm{MHz}{ }^{1} \mathrm{H}$ NMR spectroscopy characterisation of the complex metabolic pattern of urine from patients with inborn errors of metabolism: 2-Hydroxyglutaric aciduria and maple syrup urine disease. J Pharm Biomed Anal. 1997; 15:1647-1659.

38. Wishart DS, Jewison T, Guo AC, Wilson M, Knox C, Liu Y, Djoumbou Y, Mandal R, Aziat F, Dong E, Bouatra S, Sinelnikov I, Arndt D, et al. HMDB 3.0-The human metabolome database in 2013. Nucleic Acids Res. 2013; 41:D801-D807.

39. Li JQ, Zhao ZH, Feng JH, Gao JH, Chen Z. Understanding the metabolic fate and assessing the biosafety of $\mathrm{MnO}$ nanoparticles by metabonomic analysis. Nanotechnology. 2013; 24:455102.

40. Chang CC, Lin CT. LIBSVM: A library for support vector machines, ACM T Intell Syst Tech. 2011; 2:1-27.

41. Vapnik VN, "Methods of Pattern Recognition". In: J. Michael LF, Jerald LL, Steffen N, Vijay editors. The Nature of Statistical Learning Theory. New York, USA. Springer-Verlag. 1995; 138-167. 